\title{
Triple therapy avoids need for radiation in children with ALL
}

Cranial irradiation in cancer treatment can increase the risk of brain tumours and is associated with cognitive deficits. Therefore, omitting radiation might help to minimize adverse effects in the treatment of children with acute lymphoblastic leukaemia (ALL). In turn, triple intrathecal therapy has been shown to be successful at protecting the central nervous system (CNS) in these patients.

Traumatic lumbar puncture (TLP) may introduce blasts into the cerebrospinal fluid leading to a higher risk of relapse in children with ALL. Liang and coauthors wanted to determine if delaying triple intrathecal therapy until blast cells have disappeared could enable cranial irradiation to be omitted for all risk groups in paediatric ALL. Liang explains, "the first triple intrathecal therapy is delayed until disappearance of blood blasts to avoid traumatic tap with blasts (a complication associated with TLP) and to decrease frequency of CNS-3 status." The researchers present the long-term treatment outcomes of this approach, and showed that abolishing the occurrence of TLP with blasts using multidrug induction therapy can eliminate blast cells from peripheral blood. In addition, by using a modified CNS-directed therapy with no cranial irradiation, adverse effects were reduced. Impressively, radiation could be omitted from all risk groups (including very high risk) in the context of delivering effective systemic intrathecal therapy.

In conclusion, delaying triple intrathecal therapy until after the clearance of circulating blast cells can decrease CNS relapse in children with newly diagnosed ALL. In the future, Liang's team "hope that other investigators in the world may try this method and may improve it further."

\section{Lisa Hutchinson}

Original article Liu, H.-C. et al. Triple intrathecal therapy alone with omission of cranial radiation in children with acute lymphoblastic leukemia. J. Clin. Oncol. doi:10.1200/ JC0.2013.54.5020 